\title{
DISPOSICIÓN DE PLANTAS DE TOMATE EN DOSELES EN FORMA DE ESCALERA BAJO DOS DENSIDADES DE POBLACIÓN
}

\author{
ARRANGEMENT OF TOMATO PLANTS IN LADDER-SHAPED \\ CANOPIES UNDER TWO POPULATION DENSITIES
}

\author{
Felipe Sánchez-Del Castillo, Esaú del C. Moreno-Pérez*, \\ Omar A. Pastor-Zarandona y Efraín Contreras-Magaña
}

Departamento de Fitotecnia, Universidad Autónoma Chapingo. km 38.5 Carretera México-Texcoco. 56230, Chapingo, Estado de México, México.

*Autor para correspondencia (esaump10@yahoo.com.mx)

\section{RESUMEN}

El manejo de la arquitectura de la planta es de suma importancia en sistemas de producción intensiva. El objetivo del presente estudio fue evaluar el crecimiento y rendimiento de plantas de tomate (Solanum lycopersicum L.) bajo un sistema de producción alternativo basado en el despunte temprano de las yemas terminales para dejar tres racimos por planta y alta densidad de población, en condiciones hidropónicas y bajo invernadero. Se compararon tres disposiciones de plantas con doseles en forma de escalera (escaleriformes) y una cuarta con dosel uniforme (testigo). Se usaron plantas de tomate Pik Ripe 461 de hábito de crecimiento determinado. Dentro de cada sistema de producción se probaron dos densidades de población (10 y 12.5 plantas $\mathrm{m}^{-2}$ ). Se utilizó un diseño de parcelas divididas en bloques al azar con cuatro repeticiones. En las parcelas grandes se evaluaron los sistemas de producción y en las subparcelas las densidades de población. Se analizaron características morfológicas y variables de rendimiento. Se encontró que las plantas con arreglos de dosel escaleriforme rindieron entre 18 y $21 \%$ más por unidad de superficie que las de dosel uniforme, debido a que produjeron mayor cantidad de frutos sin una disminución significativa de su peso medio. La densidad de población con que se logró el mayor rendimiento $(31 \mathrm{~kg}$ $\mathrm{m}^{-2}$ ) fue de 12.5 plantas $\mathrm{m}^{-2}$. El sistema de manejo propuesto fue efectivo para incrementar el rendimiento de tomate por unidad de superficie bajo condiciones de invernadero.

Palabras clave: Solanum lycopersicum, dosel escaleriforme, arreglo de plantas, hidroponía, invernadero.

\section{SUMMARY}

The management of plant architecture is of utmost importance under intensive production systems. The aim of the present study was to evaluate growth and yield in tomato (Solanum lycopersicum L.) plants under an alternative production system based on early pruning of the terminal buds to leave just three clusters per plant and high population density under hydroponics and greenhouse conditions. Three arrangements of plants with ladder-shaped canopies and a fourth one with uniform canopy (control) were compared. Pik Ripe 461 tomato plants with determinate growth habit were used. Within each production system, two population densities (10 and 12.5 plants $\mathrm{m}^{-2}$ ) were tested. A split plot arrangement under a randomized complete blocks design with four replications was used. Production systems were evaluated in the main plots and population densities in the sub-plots. Morphological and yield-related traits were analyzed. It was found that plants with ladder-shaped canopies yielded between 18 and $21 \%$ more per unit area than those with uniform canopy, because they produced more fruits without a significant decrease in their mean weight. The population density with the highest yield $\left(31 \mathrm{~kg} \mathrm{~m}^{-2}\right)$ was 12.5 plants $\mathrm{m}^{-2}$. The proposed management system was effective to increase tomato yield per unit area under greenhouse conditions.

Index words: Solanum lycopersicum, ladder-shaped canopy, arrangement of plants, hydroponic culture, greenhouse.

\section{INTRODUCCIÓN}

El sistema de cultivo de tomate (Solanum lycopersicum L.) en invernadero que más se ha utilizado a nivel comercial en México consiste en el uso de variedades de hábito indeterminado, en densidades de población de 2 a 3 plantas $\mathrm{m}^{-2}$, donde los tallos de las plantas se dejan crecer hasta más de $7 \mathrm{~m}$ de longitud, para cosechar 15 o más racimos por planta, en un solo ciclo de cultivo por año. En condiciones hidropónicas bajo este sistema de manejo se pueden alcanzar rendimientos de 500 t ha $^{-1}$ año-1 con invernaderos de alta tecnología que permitan un excelente control de las condiciones ambientales (Peet y Welles, 2005; Resh, 2004). Aunque se puede obtener alto rendimiento, es importante resaltar que el ciclo de cultivo es muy largo (10 a 11 meses), con un periodo de inicio a fin de cosecha de seis a siete meses; además, es de difícil manejo técnico en cuanto a las prácticas culturales a realizar y a la prevención y control de plagas y enfermedades; todo ello deriva en un alto costo de producción (Méndez-Galicia et al., 2005).

Por la alta tecnología requerida para la producción de tomate bajo este sistema de cultivo, resulta poco accesible y de baja rentabilidad económica para los pequeños y medianos productores, que representan el $98 \%$ del total de las empresas (Vázquez-Rodríguez et al., 2007). Alternativamente se ha propuesto un sistema para conducir las plantas a un solo tallo y realizar despuntes tempranos para dejar sólo tres racimos en cada una. Así, el ciclo desde trasplante hasta fin de cosecha se acorta de 10 o más meses a un periodo de 3 a 4 meses (según la edad de la planta al trasplante), y en un esquema de producción continua se pueden obtener de tres a cuatro ciclos por año 
y, por lo tanto, mayor productividad anual que en los sistemas convencionales (Sánchez-Del Castillo et al., 2009; Sánchez-Del-Castillo et al., 2010; Vázquez-Rodríguez et al., 2007). La principal ventaja de este sistema es que, por lo concentrado del periodo, la cosecha se puede programar para obtenerla en los periodos cuando los precios del producto son mayores, lo que otorga el máximo beneficio económico para el productor (Sánchez-Del-Castillo et al., 2012).

Debido a la poca altura de las plantas, lograda con este sistema de manejo, el menor rendimiento por planta en un ciclo de cultivo se compensa parcialmente por el establecimiento de altas densidades de población (8 a 10 plantas $\mathrm{m}^{-2}$ ), lo cual es posible porque, con el despunte temprano, cada planta forma menor área foliar (Sánchez-Del Castillo y Ponce-Ocampo, 1998).

En este sistema generalmente se manejan camas de cultivo de 1 a $1.2 \mathrm{~m}$ de ancho, en distribución de cuatro hileras de plantas separadas por pasillos de 0.5 a $0.6 \mathrm{~m}$ de ancho. Al respecto, Sánchez-Del-Castillo et al. (2012) mencionan que los rendimientos logrados han sido altos, con promedio de $16 \mathrm{~kg} \mathrm{~m}^{-2}$ por ciclo de tres a cuatro meses (potencialmente 500 a $600 \mathrm{t} \mathrm{ha}^{-1} \mathrm{año}^{-1}$ ); se ha observado, tanto de manera experimental como en experiencias comerciales, que las plantas que se ubican en las dos hileras centrales tienen rendimientos inferiores en un 20 a $30 \%$ con respecto a los de las plantas ubicadas en las hileras de las orillas, que tienen el beneficio de recibir más radiación solar debido al espacio que hay entre camas contiguas (Sánchez-Del-Castillo et al., 2014; Ucan et al., 2005).

Para buscar una solución al sombreado entre plantas, e incrementar los rendimientos por unidad de superficie, se han realizado experimentos para comprobar que es posible distribuir homogéneamente la radiación solar sobre las hileras centrales de plantas mediante su acomodo a diferentes niveles o alturas, para generar un dosel en forma de escalera (Méndez-Galicia et al., 2005; Sánchez-Del Castillo et al., 2009; Sánchez-Del Castillo et al., 2010; Sánchez-DelCastillo et al., 2014). Se ha logrado con ello una distribución homogénea de la radiación fotosintéticamente activa entre las distintas hojas que conforman el dosel, para permitir un aumento de la tasa de asimilación neta (TAN) para un índice de área foliar (IAF) determinado, o bien incrementar el IAF aumentando la densidad de población, sin una disminución significativa de la TAN, situación que puede conducir a un incremento en la tasa de crecimiento del cultivo y en el rendimiento por unidad de superficie (Sánchez-Del Castillo et al., 2009).

En sistemas escaleriformes, formados con contenedores (tinas de cultivo) a diferentes alturas, se han probado diferentes densidades de población para plantas despuntadas a tres racimos, hasta 24 plantas $\mathrm{m}^{-2}$ útil (Méndez-Galicia et al., 2005; Sánchez-Del Castillo et al., 2009; Sánchez-Del Castillo et al., 2010; Sánchez-Del-Castillo et al., 2014). Aunque las densidades altas han permitido altos rendimientos (hasta $30 \mathrm{~kg} \mathrm{~m}^{-2}$ en un ciclo de menos de 4 meses), la competencia entre plantas de las mismas hileras, sobre todo con cultivares de hábito indeterminado, que producen tres hojas entre racimos, ha ocasionado una disminución importante en el peso medio de fruto, lo que afecta de manera negativa su precio en el mercado, sobre todo para tomates tipo bola. Por ello, en el presente estudio, un primer objetivo fue encontrar la densidad de población más adecuada en rendimiento y tamaño de fruto con estos sistemas, mediante un cultivar de hábito determinado tipo bola, que presenta menos área foliar por planta al tener sólo una hoja entre racimos.

Los sistemas escaleriformes de producción más exitosos en cuanto a rendimiento por unidad de superficie han sido los propuestos por Méndez-Galicia et al. (2005); Sánchez-Del Castillo et al. (2009) y Sánchez-Del-Castillo et al. (2014); sin embargo, dichos sistemas están basados en hileras de plantas de cuatro o tres niveles de altura, que generan como inconvenientes a una escala comercial el elevado costo de los contenedores, de las estructuras necesarias para sostenerlos en los distintos niveles y de la mano de obra para su instalación; por ello, en el presente trabajo se propuso como un segundo objetivo comparar el crecimiento y rendimiento de estos sistemas contra el de otro arreglo escaleriforme de sólo dos niveles, que permite el uso de contenedores más fáciles de implementar en la práctica y que tienen un costo de instalación mucho más bajo.

\section{MATERIALES Y MÉTODOS}

\section{Sitio experimental}

La presente investigación se llevó a cabo de febrero a junio de 2014, en un invernadero con cubierta de polietileno y estructura metálica en el Campo Experimental de la Universidad Autónoma Chapingo, ubicado en Chapingo, Estado de México, a $19^{\circ} 29^{\prime}$ N, $98^{\circ} 53^{\prime}$ O y altitud de 2250 msnm. Además de tener ventanas protegidas con malla antiáfidos y cortinas enrollables, el invernadero estaba equipado con un sistema de muro húmedo y extractores, y un sistema de calefacción que en conjunto permitían un adecuado control de la temperatura.

\section{Material genético}

Se utilizó el híbrido comercial Pik Ripe 461 de hábito de crecimiento determinado y fruto tipo bola, que es apreciado 
en el mercado por sus frutos grandes, color uniforme, buen sabor, firmeza y relativamente larga vida de anaquel (Sánchez-Del Castillo et al., 2010). Este híbrido es comercializado por la empresa Seminis y es resistente a los virus Rizado Amarillo del Tomate y del Mosaico del Tomate, además de cáncer de tallo por Alternaria, Verticilium sp, mancha gris de la hoja causada por Stemphylium solani y Fusarium oxisporium f. sp. (Seminis, 2016).

\section{Tratamientos, diseño y unidad experimental}

Se establecieron ocho tratamientos como resultado de la combinación de cuatro formas de disposición de las hileras de plantas y dos densidades de población. La densidad denominada baja se ajustó a 10 plantas $\mathrm{m}^{-2}$ y la alta a 12.5 plantas $\mathrm{m}^{-2}$; para ambos casos se consideraron pasillos de $0.6 \mathrm{~m}$ de ancho.

Los tratamientos 1 y 2 fueron en sistemas de escalera simple en baja y alta densidad, respectivamente. Estos consistieron de unidades experimentales formadas por cuatro contenedores (tinas de lámina galvanizada) de $1 \mathrm{~m}$ de largo por $25 \mathrm{~cm}$ de ancho y $25 \mathrm{~cm}$ de profundidad cada uno y se rellenaron con arena de tezontle rojo con partícu- las de 2 a $4 \mathrm{~mm}$ de diámetro como sustrato y se colocaron con una orientación este-oeste a diferentes alturas $(30 \mathrm{~cm}$ de desnivel entre ellas), quedando la tina inferior del lado sur y la más alta del lado norte. El ancho de la escalera fue de $1 \mathrm{~m}$ (Figura $1 \mathrm{~A}$ ).

Los tratamientos 3 y 4 fueron denominados sistemas de doble escalera en punta con baja y alta densidad, respectivamente. Estos consistieron de unidades experimentales formadas por cinco contenedores de $1 \mathrm{~m}$ de largo por 25 $\mathrm{cm}$ de ancho y $25 \mathrm{~cm}$ de profundidad; los contenedores se colocaron con orientación norte-sur, en forma de doble escalera de $1.25 \mathrm{~cm}$ de ancho en tres niveles de altura con $30 \mathrm{~cm}$ de desnivel cada escalón (Figura 1 B).

Los tratamientos 5 y 6 se denominaron como sistemas de doble escalera plana en baja y alta densidad, respectivamente. Estos consistieron de unidades experimentales formadas por cuatro contenedores de $1 \mathrm{~m}$ de largo por 25 $\mathrm{cm}$ de ancho y $25 \mathrm{~cm}$ de profundidad, colocadas con orientación norte-sur, las cuales ocuparon $1 \mathrm{~m}$ de ancho y se formaron con dos escalones de cada lado con $30 \mathrm{~cm}$ de desnivel entre cada escalón (Figura 1 C).

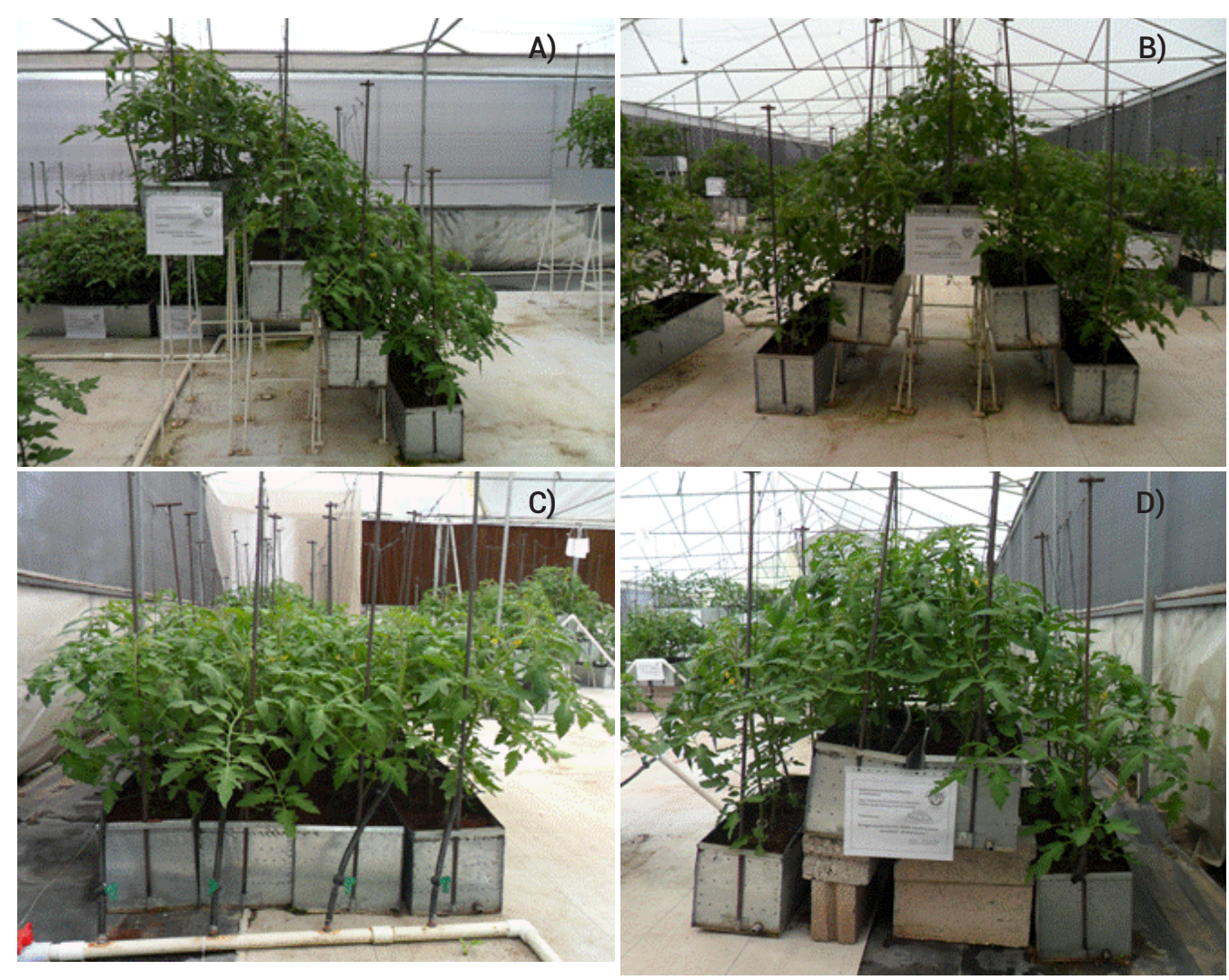

Figura 1. Tratamientos con diferentes disposiciones de plantas: A) Dosel en escalera simple en dirección este-oeste. B) Dosel en escalera doble en punta en dirección norte-sur. C) Dosel en escalera plana en dirección norte-sur. D) Dosel uniforme en dirección norte-sur. 
Los tratamientos 7 y 8 se denominaron sistemas de dosel uniforme en baja y alta densidad, respectivamente. Las unidades experimentales consistieron de cuatro contenedores de $1 \mathrm{~m}$ de largo por $25 \mathrm{~cm}$ de ancho y $25 \mathrm{~cm}$ de profundidad, colocados al mismo nivel con orientación norte-sur, y ocuparon $1 \mathrm{~m}$ de ancho (Figura 1 D).

El experimento fue establecido bajo un arreglo factorial de parcelas divididas. En las parcelas grandes se colocaron los arreglos escaleriformes en diseño de bloques completos al azar con cuatro repeticiones y las parcelas chicas fueron las densidades de población. El tamaño de la unidad experimental de parcela chica fue de $1 \mathrm{~m}^{2}$ útil, excepto para el tratamiento de doble escalera en punta que fue de $1.25 \mathrm{~m}^{2}$ útiles.

\section{Manejo del experimento}

La siembra se realizó en charolas germinadoras de poliestireno de 60 cavidades con capacidad de $180 \mathrm{~cm}^{3}$ cada una, utilizando como sustrato una mezcla de turba y perlita en proporción 1:1 (v:v). A los 35 días después de la siembra (dds) se hizo el trasplante. Durante todo el ciclo del cultivo se usó la solución nutritiva propuesta por Sánchez-del Castillo et al. (2009), cuya concentración de nutrimentos minerales en $\mathrm{mg}$ por litro ( $\mathrm{mg} \mathrm{L}^{-1}$ ) fue la siguiente: $\mathrm{N}=250$, $P=60, K=250, \mathrm{Ca}=300, \mathrm{~S}=200, \mathrm{Mg}=60, \mathrm{Fe}=3, \mathrm{Mn}=0.5$, $\mathrm{Cu}=0.1, \mathrm{~B}=0.1$ y $\mathrm{Zn}=0.1$. Como fuentes de estos elementos se emplearon los fertilizantes comerciales nitrato de calcio, sulfato de potasio, ácido fosfórico al $85 \%$, sulfato de magnesio, sulfato ferroso, sulfato de manganeso, tetraborato de sodio (bórax), sulfato de cobre y sulfato de zinc.

El trasplante se hizo en las tinas metálicas rellenas de tezontle rojo con partículas de 1 a $4 \mathrm{~mm}$ de diámetro; posteriormente, las plantas fueron tutoradas individualmente con cordones de rafia. Los brotes laterales se podaron cuando tenían una longitud de $10 \mathrm{~cm}$ o menos, de tal forma que las plantas crecieran a un solo tallo. En todos los tratamientos las plantas fueron despuntadas (eliminación de la yema terminal) a dos hojas por encima de la tercera inflorescencia, para dejar sólo tres racimos por cada planta y detener su crecimiento a menos de $1 \mathrm{~m}$ de altura.

El sistema de riego fue por goteo, sin recuperación de la solución nutritiva. En este se colocaron cuatro emisores por tina, con lo cual se proporcionaron un promedio de cuatro riegos al día con la solución antes mencionada. El primer corte se realizó a los 101 dds y el último a los 140 dds.

\section{Variables evaluadas y análisis estadístico}

Las variables que se evaluaron fueron: 1) altura de planta en cm, a los 65 días después de la siembra (dds); 2) diámetro de tallo en cm a los 65 dds, medido debajo del entrenudo inmediatamente inferior al primer racimo; 3) área foliar por planta en $\mathrm{cm}^{2}$ e Indicie de área foliar $\left(\mathrm{m}^{2} \mathrm{~m}^{-2}\right)$ a los 65 dds, para ello se tomó una muestra de 100 hojas, a las que se les midió el largo, ancho y área foliar de cada una mediante un medidor de área foliar (LI-3100, LI-CORß, Lincoln, Nebraska, USA).

Con el fin de obtener una función que pudiera estimar el área foliar de manera no destructiva, tal y como lo mencionan Suazo-López et al. (2014), se usó una regresión lineal en donde la variable independiente $(X)$ fue el producto de la multiplicación del largo por el ancho de cada hoja y la variable dependiente $(Y)$ fue el área foliar de cada una de ellas. La función obtenida $(Y=0.2639 X+29.334)$ tuvo una $\mathrm{R}^{2}$ de 0.96; en la misma fecha se midió el largo y ancho de todas las hojas de una planta por hilera para cada tratamiento y repetición; y con estos datos y la función obtenida anteriormente se estimó el área foliar por planta y el índice de área foliar ( $\mathrm{m}^{2}$ de hoja / $\mathrm{m}^{2}$ de superficie cubierta); 4) rendimiento por planta ( $\mathrm{kg} /$ planta); 5) rendimiento por unidad de superficie ( $\mathrm{kg} \mathrm{m}^{-2}$ de invernadero); 6) número de frutos por planta; y 7) número de frutos por $\mathrm{m}^{-2}$ de invernadero).

Los datos obtenidos se sometieron a la prueba de análisis de varianza y comparación de medias de Tukey $(P \leq$ 0.05).

\section{RESULTADOS Y DISCUSIÓN}

\section{Variables morfológicas}

Los análisis de varianza para las variables morfológicas evaluadas a los 65 dds (Cuadro 1) mostraron diferencias significativas entre los arreglos de dosel para el diámetro de tallo, y diferencias altamente significativas en altura de planta (AP), área foliar por planta e índice de área foliar (IAF) por unidad de superficie, mientras que para la densidad de población hubo diferencia significativa entre AP y altamente significativa en IAF. La interacción arreglo de dosel por densidad no fue significativa para ninguna de las variables.

En el Cuadro 2 se observa que, en el promedio de las densidades, las plantas de mayor altura fueron las de dosel uniforme y aunque resultaron estadísticamente iguales a las de los doseles de doble escalera plana y doble escalera en punta, muestran tendencia a ser diferentes, mientras que las de menor altura fueron las del arreglo en escalera simple (12\% menor a las del testigo uniforme). Esto podría explicarse porque las hileras centrales de plantas en disposición uniforme quedan más sombreadas al tener 
Cuadro 1. Cuadrados medios y niveles de significancia para variables morfológicas a los 65 días después de la siembra.

\begin{tabular}{lccccc}
\hline Fuente de variación & GL & Altura de planta & Diámetro de tallo & Área foliar por planta & Índice de área foliar \\
\hline Arreglo & 3 & $204 * *$ & $0.0080 *$ & $3,861,956 * *$ & $5.54 * *$ \\
Repetición & 3 & 21 & 0.0036 & 91,937 & 0.12 \\
Error a & 9 & 30 & 0.0058 & 82,649 & 0.10 \\
Densidad & 1 & $128 *$ & $0.004 \mathrm{~ns}$ & $1815 \mathrm{~ns}$ & $9.16 * *$ \\
ArregloxDensidad & 3 & $30 \mathrm{~ns}$ & $0.0009 \mathrm{~ns}$ & $51,758 \mathrm{~ns}$ & $0.16 \mathrm{~ns}$ \\
Error b & 12 & 35 & 0.0048 & 65,080 & 0.08 \\
Total & 31 & & & & 5.96 \\
CV (\%) & & 5.76 & 3.5 & 5.97 &
\end{tabular}

*: Significancia a P $\leq 0.05$; **: Significancia a P $\leq 0.01$; ns: no significativo; CV: coeficiente de variación.

Cuadro 2. Comparación de medias de características morfológicas de tomate a los 65 días después de la siembra en función de la disposición de las plantas.

\begin{tabular}{lcccc}
\hline Disposición & $\begin{array}{c}\text { Altura de la planta } \\
(\mathrm{cm})\end{array}$ & $\begin{array}{c}\text { Diámetro de tallo } \\
(\mathrm{cm})\end{array}$ & $\begin{array}{c}\text { Área foliar por planta } \\
\left(\mathrm{cm}^{2}\right)\end{array}$ & $\begin{array}{c}\text { Índice de área foliar } \\
\left(\mathrm{m}^{2} \mathrm{~m}^{-2}\right)\end{array}$ \\
\hline Doble escalera plana & $101.2 \mathrm{ab}$ & $1.16 \mathrm{a}$ & $3898 \mathrm{c}$ & $4.3 \mathrm{c}$ \\
Doble escalera punta & $102.7 \mathrm{ab}$ & $1.14 \mathrm{ab}$ & $4388 \mathrm{~b}$ & $5.3 \mathrm{~b}$ \\
Escalera simple & $97.8 \mathrm{~b}$ & $1.15 \mathrm{a}$ & $3595 \mathrm{c}$ & $4.0 \mathrm{c}$ \\
Dosel uniforme & $109.8 \mathrm{a}$ & $1.09 \mathrm{~b}$ & $5187 \mathrm{a}$ & $5.8 \mathrm{a}$ \\
DSH (0.05) & 8.8 & 0.059 & 378 & 0.43 \\
\hline
\end{tabular}

Medias con letras iguales en cada columna, no son estadísticamente diferentes (Tukey, 0.05).

competencia completa, lo que seguramente estimula su elongación por favorecerse la síntesis de auxinas (Taiz y Zeiger, 2006).

Las plantas con menor diámetro de tallo, mayor área foliar y mayor IAF fueron las del arreglo de dosel uniforme. El menor diámetro de tallo también fue consecuencia del sombreado por la competencia completa, lo que afecta el promedio del grosor de las plantas de todo el tratamiento. En cuanto a la mayor área foliar por planta e IAF, se puede explicar, como lo señalan Gardner et al. (1990) y Taize y Zeiger (2006) en el sentido de que las hojas que crecen en condición de menor incidencia de radiación fotosintéticamente activa tienden a crecer más en área, pero presentan su limbo con menos grosor (menos capas de células), en tanto que las que reciben una mayor cantidad de radiación solar, como es el caso de las plantas de los arreglos escaleriformes, las hojas son más gruesas, pero de menor superficie; es decir, tienen mayor peso foliar específico, lo que según Björkman (1981) resulta en mayor tasa de fotosíntesis por unidad de área. Estos resultados coinciden con los encontrados por Sánchez-del Castillo et al. (2010), quienes reportaron que el IAF en dosel uniforme de cuatro hileras fue superior al del dosel escaleriforme. Los arreglos doble escalera plana y escalera simple fueron los que obtuvieron menor área foliar por planta y menor IAF.
Entre densidades de población no se encontraron diferencias significativas para las variables diámetro de tallo, área foliar por planta y altura de planta (Cuadro 3). Resultados similares encontraron Sánchez-del Castillo et al. (2009) al comparar tres densidades de población en doseles escaleriformes; pero la densidad de 12.5 plantas $\mathrm{m}^{-2}$ obtuvo mayor IAF que la de 10 plantas $\mathrm{m}^{-2}$ (Cuadro 3), esto como consecuencia de que la mayor densidad de población no se reflejó en una disminución significativa del área foliar por planta.

\section{Rendimiento y sus componentes}

El análisis de varianza de rendimiento y sus componentes (Cuadro 4) indican que hubo diferencias significativas tanto entre arreglos de las plantas, como entre densidades de población para todas las variables, excepto para el peso medio de fruto. En ningún caso la interacción arreglo por densidad fue significativa.

En el Cuadro 5 se observa que el rendimiento por planta y por unidad de superficie fueron estadísticamente menores para el arreglo de dosel uniforme que para los escaleriformes, ya que en estos últimos el rendimiento por unidad de superficie aumentó de 18 a $21 \%$. Cabe señalar que los rendimientos obtenidos para cada tratamiento se lograron 
Cuadro 3. Comparación de medias para características morfológicas de tomate a los 65 días después de la siembra en función de la densidad de población.

\begin{tabular}{lcccc}
\hline $\begin{array}{c}\text { Densidad de población } \\
\left(\text { plantas } \mathrm{m}^{-2}\right)\end{array}$ & Altura de planta $(\mathrm{cm})$ & Diámetro de tallo $(\mathrm{cm})$ & $\begin{array}{c}\text { Área foliar por planta } \\
\left(\mathrm{cm}^{2}\right)\end{array}$ & $\begin{array}{c}\text { Índice de área foliar } \\
\left(\mathrm{m}^{2} \mathrm{~m}^{-2}\right)\end{array}$ \\
\hline 10 & $100.9 \mathrm{a}$ & $1.13 \mathrm{a}$ & $4274 \mathrm{a}$ & $4.3 \mathrm{a}$ \\
12.5 & $104.9 \mathrm{a}$ & $1.14 \mathrm{a}$ & $4259 \mathrm{a}$ & $5.4 \mathrm{~b}$ \\
$\mathrm{DSH}(0.05)$ & 4.5 & 0.03 & 196 & 0.25 \\
\hline
\end{tabular}

Medias con letras iguales en cada columna no son estadísticamente diferentes (Tukey, 0.05).

Cuadro 4. Cuadrados medios y niveles de significancia para variables morfológicas a los 65 días después de la siembra.

\begin{tabular}{lcccccc}
\hline Fuente de variación & $\mathrm{GL}$ & $\begin{array}{c}\text { Rendimiento } \\
(\mathrm{kg} / \mathrm{planta})\end{array}$ & $\begin{array}{c}\text { Rendimiento } \\
\left(\mathrm{kg} \mathrm{m}^{-2}\right)\end{array}$ & $\begin{array}{c}\text { Frutos por } \\
\text { planta }\end{array}$ & $\begin{array}{c}\text { Frutos por } \\
\mathrm{m}^{2}\end{array}$ & $\begin{array}{c}\text { Peso medio de } \\
\text { fruto }(\mathrm{g})\end{array}$ \\
\hline Arreglo & 3 & $0.325 * *$ & $50.47 * *$ & $10.58 * *$ & $1697 *$ & $10.84 \mathrm{~ns}$ \\
Repetición & 3 & 0.012 & 1.63 & 0.03 & 4.03 & 55.31 \\
Error a & 9 & 0.007 & 0.98 & 0.37 & 48.05 & 8.36 \\
Densidad & 1 & $0.107 * *$ & $200.1 * *$ & $3.25 * *$ & $6412 * *$ & $0.36 \mathrm{~ns}$ \\
Arreglo x Densidad & 3 & $0.0006 \mathrm{~ns}$ & $0.31 \mathrm{~ns}$ & $0.09 \mathrm{~ns}$ & $42.19 \mathrm{~ns}$ & $30.59 \mathrm{~ns}$ \\
Error b & 12 & 0.003 & 0.32 & 0.18 & 24.26 & 20.86 \\
Total & 31 & & & & & 2.08 \\
CV $(\%)$ & & 4.13 & 3.02 & 3.05 & 3.08 & 2.56 \\
\hline
\end{tabular}

*: Significancia a P $\leq 0.05 ; * *$ : Significancia a P $\leq 0.01$; ns: no significativo; CV: coeficiente de variación.

Cuadro 5. Medias de rendimiento y sus componentes en tomate en función de la disposición de las plantas.

\begin{tabular}{lccccc}
\hline Disposición de plantas & $\begin{array}{c}\text { Rendimiento } \\
\left(\mathrm{kg} \mathrm{planta}^{-1}\right)\end{array}$ & $\begin{array}{c}\text { Rendimiento } \\
\left(\mathrm{kg} \mathrm{m}^{-2}\right)\end{array}$ & Frutos por planta & Frutos por $\mathrm{m}^{2}$ & Peso de fruto $(\mathrm{g})$ \\
\hline Doble escalera plana & $2.64 \mathrm{a}$ & $29.68 \mathrm{ab}$ & $14.97 \mathrm{a}$ & $168.12 \mathrm{a}$ & $176.76 \mathrm{a}$ \\
Doble escalera punta & $2.47 \mathrm{~b}$ & $30.0 \mathrm{a}$ & $13.97 \mathrm{~b}$ & $169.37 \mathrm{a}$ & $177.18 \mathrm{a}$ \\
Escalera simple & $2.59 \mathrm{a}$ & $29.15 \mathrm{~b}$ & $14.51 \mathrm{ab}$ & $163.12 \mathrm{a}$ & $179.37 \mathrm{a}$ \\
Dosel uniforme & $2.19 \mathrm{c}$ & $24.64 \mathrm{c}$ & $12.33 \mathrm{c}$ & $138.25 \mathrm{~b}$ & $178.2 \mathrm{a}$ \\
DSH (0.05) & 0.07 & 0.85 & 0.63 & 7.31 & 8.68 \\
\hline
\end{tabular}

Medias con letras iguales en cada columna no son estadísticamente diferentes (Tukey, 0.05).

en un periodo de $105 \mathrm{~d}$ del trasplante a fin de cosecha. Resultados similares encontraron Vázquez-Rodríguez et al. (2007), Sánchez-del Castillo et al. (2010) y Sánchez-DelCastillo et al. (2014), al oservar que los doseles escaleriformes obtuvieron mayor rendimiento por unidad de superficie que el dosel uniforme.

El rendimiento por unidad de superficie del arreglo de doble escalera en punta resultó estadísticamente igual al dosel de doble escalera plana. En cuanto al número de frutos por planta el dosel de doble escalera plana y el dosel de escalera simple también resultaron estadísticamente iguales. La menor cantidad de frutos por planta se obtuvo con el dosel uniforme, lo que seguramente se debió a que los arreglos escaleriformes recibieron una distribución más homogénea de la luz en las hojas del dosel, en tanto que en las hileras de las plantas con disposición de dosel uniforme la radiación solar es casi totalmente interceptada por las hojas superiores debido a que sus plantas se encontraban en competencia completa y alta densidad de población (Heuvelink y Dorais, 2005; Papadopoulos y Ormrod, 1990; Sánchez-Del-Castillo et al., 2014; Ucan et al., 2005).

Cabe destacar que el rendimiento por unidad de superficie en el arreglo de dosel uniforme fue más alto (Cuadro 5) con respecto a otros experimentos similares (SánchezDel Castillo et al., 2010; Vázquez-Rodríguez et al., 2007), lo que se puede explicar por la mayor densidad de población utilizada en el presente experimento. Ya se había establecido como densidad óptima 8 plantas $\mathrm{m}^{-2}$ para el sistema de dosel uniforme manejado a tres racimos por planta (Sánchez y Corona, 1994; Sánchez-Del Castillo y Ponce-Ocampo, 1998), pero se habían utilizado cultivares 
Cuadro 6. Medias de rendimiento y sus componentes en tomate en función de la densidad de población.

\begin{tabular}{lccccc}
\hline $\left.\begin{array}{l}\text { Densidad de población } \\
(\text { plantas m }\end{array}\right)$ & $\begin{array}{c}\text { Rendimiento } \\
\left(\mathrm{kg} \mathrm{planta}^{-1}\right)\end{array}$ & $\begin{array}{c}\text { Rendimiento } \\
\left(\mathrm{kg} \mathrm{m}^{-2}\right)\end{array}$ & Frutos por planta & Frutos por $\mathrm{m}^{2}$ & Peso de fruto (g) \\
\hline 10 & $2.53 \mathrm{a}$ & $25.87 \mathrm{~b}$ & $14.3 \mathrm{a}$ & $145 \mathrm{~b}$ & $180 \mathrm{a}$ \\
12.5 & $2.42 \mathrm{~b}$ & $30.86 \mathrm{a}$ & $13.6 \mathrm{~b}$ & $174 \mathrm{a}$ & $178 \mathrm{a}$ \\
$\mathrm{DSH}(0.05)$ & 0.04 & 0.44 & 0.32 & 3.79 & 3.51 \\
\hline
\end{tabular}

Medias con letras iguales en cada columna no son estadísticamente diferentes (Tukey, 0.05).

de hábito indeterminado, los cuales presentan mayor densidad de follaje que los determinados debido a un mayor número de hojas por planta.

Los arreglos con mayor densidad de población tuvieron menor rendimiento por planta, pero mayor rendimiento por unidad de superficie (19\% mayor a los de menor densidad), con diferencias estadísticamente significativas (Cuadro 6). No se encontraron diferencias para el tamaño de fruto entre densidades, lo que indica que se puede utilizar comercialmente la densidad alta para incrementar el rendimiento.

Estos resultados sugieren que se deben realizar otros ensayos encaminados a establecer una densidad óptima para cultivares de tomate de hábito determinado, los cuales ofrecen la posibilidad de mayor rendimiento por unidad de superficie que los de crecimiento indeterminado, que son los que están actualmente en uso en invernaderos.

Los menores valores obtenidos en rendimiento y sus componentes por el arreglo de dosel uniforme se explican en función de que bajo este arreglo se da una mayor competencia entre las plantas de las hileras centrales por la radiación incidente con respecto a los arreglos escaleriformes donde, como ya se señaló, se logra una distribución más uniforme de la radiación en las hojas de todo el dosel.

El resultado de un rendimiento por unidad de superficie del orden de $30 \mathrm{~kg} \mathrm{~m}^{-2}$ en un ciclo de $107 \mathrm{~d}$ de trasplante a cosecha es importante desde el punto de vista agronómico ya que eso implica, aun para invernaderos de mediana tecnología, el potencial de obtener comercialmente hasta $90 \mathrm{~kg} \mathrm{~m}^{-2}$ (900 t ha-1 año-1) en tres ciclos por año, lo que es aproximadamente el doble de lo que se produce en invernaderos holandeses de alta tecnología con costos de producción muy elevados (Kinet y Peet, 1997; Peet y Welles, 2005) y el triple (300 t ha-1 año-1) de lo que se obtiene en invernaderos de mediana tecnología en México en suelo o en sistemas hidropónicos (Castellanos y Borbón, 2008).

\section{CONCLUSIONES}

Para una misma densidad de población, la disposición de las plantas para formar doseles en forma de escalera produjo mayor rendimiento por unidad de superficie que la dispuesta para formar un dosel uniforme a la misma altura. Las plantas en densidad de población alta (12.5 plantas $\mathrm{m}^{-2}$ ) rindieron más por unidad de superficie que las cultivadas a menor densidad (10 plantas $\mathrm{m}^{-2}$ ) sin una disminución significativa del peso del fruto. En virtud de lo anterior, por el rendimiento obtenido y facilidad de instalación del sistema de escalera plana, se considera importante someterlo a validación a nivel comercial con productores.

\section{BIBLIOGRAFÍA}

Björkman 0. (1981) Responses to different quantum flux densities. In: Physiological Plant Ecology I: Responses to Physical Plant Environment. O. L. Lange, P. S. Nobel, C. B. Osmond and H. Ziegler (eds.). Springer-Verlag, Berlin, Germany. pp:57-107.

Castellanos J. Z. y M. C. Borbón (2008) Panorama de la horticultura protegida en México. In: Manual de Producción de Tomate en Invernadero. J. Z. Castellanos (ed.). INTAGRI. México. pp:1-18.

Gardner F. P., R. B. Pearce and R. L. Mitchell (1990) Physiology of Crop Plants. lowa State University Press. Ames, lowa, USA. 327 p.

Heuvelink E. and M. Dorais (2005) Crop growth and yield. In: Tomatoes. E. Heuvelink (ed.). CABI Publishing. Oxfordshire, UK. pp:85-144.

Kinet J. M. and M. M. Peet (1997) Tomato. In: The Physiology of Vegetable Crops. H. C. Wien (ed.). CABI Publishing. Cambridge, UK. pp:207-258.

Méndez-Galicia T., F. Sánchez-Del Castillo, J. Sahagún-Castellanos y E. Contreras-Magaña (2005) Doseles escaleriformes con hileras de plantas de jitomate orientadas en dirección este-oeste. Revista Chapingo Serie Horticultura 11:185-192.

Papadopoulos A. P. and D. P. Ormrod (1990) Plant spacing effects on yield of the greenhouse tomato. Canadian Journal of Plant Science 70:565-573.

Peet M. M. and G. Welles (2005) Greenhouse tomato production. In: Tomatoes. E. Heuvelink (ed). CABI Publishing. Oxfordshire, UK. pp:257-304.

Resh H. M. (2004) Cultivos Hidropónicos. 3a ed. Mundi-Prensa. Madrid, España. $369 p$

Sánchez-Del-Castillo F., O. A. Bastida-Cañada, E. C. Moreno-Pérez, E. Contreras-Magaña y J. Sahagún-Castellanos (2014) Rendimiento de jitomate con diferentes métodos de cultivo hidropónico basados en doseles escaleriformes. Revista Chapingo Serie Horticultura 20:239-251

Sánchez del C. F. y T. Corona S. (1994) Evaluación de cuatro variedades de jitomate (Lycopersicum esculentum Mill.) bajo un sistema hidropónico a base de despuntes y altas densidades. Revista Chapingo Serie Horticultura 2:109-114

Sánchez-del Castillo F., E. C. Moreno-Pérez, R. Coatzín-Ramírez, M. T. ColinasLeón y A. Peña-Lomelí (2010) Evaluación agronómica y fisiotécnica de cuatro sistemas de producción en dos híbridos de jitomate. Revista Chapingo Serie Horticultura 16:207-214.

Sánchez-del-Castillo F., E. C. Moreno-Pérez and E. Contreras-Magaña (2012) Development of alternative crop systems for commercial production of vegetables in hydroponics-I: Tomato. 
Acta Horticulturae 947:179-187.

Sánchez-del Castillo F., E. C. Moreno-Pérez y E. L. Cruz-Arellanes (2009) Producción de jitomate hidropónico bajo invernadero en un sistema de dosel en forma de escalera. Revista Chapingo Serie Horticultura 15:67-73.

Sánchez-Del Castillo F. y J. Ponce-Ocampo (1998) Densidad de plantación y nivel de despunte en jitomate (Lycopersicon esculentum Mill.) cultivado en hidroponia. Revista Chapingo Serie Horticultura 4:89-93.

Seminis (2016) Pik Ripe 461. http://www.seminis.mx/product/pikripe-461/402 (Mayo 2016)

Suazo-López F., R. Zepeda-Bautista, F. Sánchez-Del-Castillo, J. J. MartínezHernández, J. Virgen-Vargas and L. Tijerina-Chávez (2014) Growth and yield of tomato (Solanum lycopersicum L.) as affected by hydroponics, greenhouse and irrigation regimes. Annual Research and Review in Biology 4:4246-4258.

Taiz L. and E. Zeiger (2006) Plant Physiology. $4^{\text {th }}$ ed. Sinauer Associates Inc. Publishers. Sunderland. Massachusetts, USA. $705 \mathrm{p}$.

Ucan C. I., F. Sánchez-del-C., E. Contreras M. y T. Corona S. (2005) Efecto de la densidad de población y raleo de frutos sobre el rendimiento y tamaño de fruto en tomate. Revista Fitotecnia Mexicana 28:3338.

Vázquez-Rodríguez J. C., F. Sánchez-Del-Castillo y E. C. Moreno-Pérez (2007) Producción de jitomate en doseles escaleriformes bajo invernadero. Revista Chapingo Serie Horticultura 13:55-62. 